\section{Durchbruch für den Strukturwandel?}

Wie kann Politik stärker als bisher Rücksicht auf ökologische Belange nehmen? Politikintegration ist hier auf deutscher wie europäischer Ebene eines der meistdiskutiertesten Instrumente. Bisher finden ökologische Folgen nur selten Eingang in Entscheidungen der Fachministerien, wohingegen soziale, vor allem aber ökonomische Folgen in der Regel einbezogen werden. Können die derzeit erarbeiteten Nachhaltigkeitsstrategien hier zu einer Veränderung beitragen?

$\mathrm{D}$ Von Annette Volkens Thema der Umweltministerien. Das Paradoxon dieser Situation: Mit der Bildung eigenständiger Ministerien sollten ökologische Belange größeres Gewicht erhalten, nachdem sie vorher in andere Ressorts, wie zum Beispiel das deutsche Innenministerium, integriert waren. Mit der geschaffenen separaten Stellung, sich verändernden Rahmenbedingungen sowie für vordringlicher gehaltenen Problemen geriet die Bedeutung der Umwelt trotz rhetorischer Unterstiutzung ins Hintertreffen. In der praktischen Umsetzung blieben die Umweltministerien auf sich allein gestellt und konnten ihre Vorstellungen einer umweltgerechteren Politik nur selten gegen andere Ressorts durchsetzen: Vor allem Wirtschafts-, Arbeits- und Sozialpolitik, sowie auf EU-Ebene zusätzlich die freie Handelspolitik, hatten Vorrang.

Mit Hilfe von Politikintegration soll sich dies ändern. Alle Fachpolitiken sollen die Folgen ihres Handelns stärker in Zusammenhang mit anderen Bereichen bringen. Politikintegration kann mittels zweier Ansätze praktisch umgesetzt werden: Durch die übergreifende Festlegung klarer Zielvorgaben und Strategien, etwa im Rahmen einer Nachhaltigkeitsstrategie, die ein Umdenken in der politischen Entscheidungsfindung erfordern. Oder durch institutionelle Veränderungen auf Ebene der politischen Entscheidungsstrukturen. Beides sind Faktoren einer erfolgreichen Politikintegration. Wichtig für die erfolgreiche Umsetzung beider Wege ist die Definition von Handlungsfeldern.

\section{- Der Cardiff-Prozess als Anstoß}

Die Notwendigkeit eines stärkeren Zusammenwirkens der Fachpolitiken wurde in der Europäischen Union (EU) erkannt und seit 1998 mit dem Cardiff-Prozess manifestiert. Der Fokus liegt hier auf der Entwicklung von Strategien für den Einbezug ökologischer Aspekte in vereinbarte Handlungsfelder (1). Die Umsetzung des Cardiff-Prozesses lässt jedoch zu wünschen übrig: Nach einer Studie im Auftrag von Umweltministerium und Umweltbundesamt sind bis Ende 2000 von den definierten acht Handlungsfeldern nur in den Bereichen Verkehr, Energie und Entwicklung annähernd gute Erfolge erzielt worden. Insbesondere die Bereiche Binnenmarkt sowie Wirtschaft und Finanzen lagen weit hinter den Anforderungen zurück. Die Bewertungen der Studie beziehen sich auf Problemanalyse, Beteiligung des Feldes an Umweltbelastungen und die Entwicklung einer Strategie zu deren Verringerung. Eine Umsetzung von Strategien ist bisher in keinem Feld erfolgt (2).

Die Politikintegration steckt auch in Deutschland noch in den Kinderschuhen. Während - insbesondere im Energiebereich - vielfältige Beispiele für widersprüchliche und kontraproduktive politische Entscheidungen auszumachen sind (3), zeigt bisher nur das im Herbst letzten Jahres verabschiedete Klimaschutzprogramm die Unumgänglichkeit eines abgestimmten Vorgehens: Durch Festlegung sektoraler Emissionsminderungs-Zielvorgaben und die Anforderung, dass wenn "ein Sektor nicht in der Lage ist sein Minderungsziel zu erreichen [...] dies durch verstärkte Anstrengungen in anderen Sektoren kompensiert werden" muss (4).

Die auf EU-Ebene vorgenommene Problemanalyse findet in Deutschland bisher weder für einzelne Handlungsfelder noch Ressorts statt. Lediglich für Luftschadstoffe wie Kohlendioxid ist die Beteiligung einzelner Handlungsfelder bzw. Sektoren an Umweltproblemen wie dem Treibhauseffekt bekannt. Eine Analyse, inwieweit zum Beispiel energiepolitische Entscheidungen des Wirtschaftsministeriums Auswirkungen auf die Umwelt besitzen und welche Maßnahmen zu deren Bekämpfung wiederum von Seiten des Umweltministeriums eingesetzt werden müssen - und welche wirtschaftlichen Folgen diese dann mit sich bringen -, erfolgt in der Regel nicht.

\section{Ein Green Cabinet zur besseren Abstimmung}

Im Gegensatz zur EU hat Deutschland - mit Ausnahme des Klimaschutzprogramms - im ersten Schritt keine Handlungsfelder für eine Politikintegration bestimmt, sondern sich zunächst für institutionelle Veränderungen entschieden: Im Sommer 2000 wurde im Rahmen der Diskussion um die Entwicklung einer Nachhaltigkeitsstrategie die Gründung des Staatssekretärsausschusses für nachbaltige Entwicklung beschlossen. Der Fokus der Arbeit liegt auf der ökologischen Säule der Nachhaltigkeit. Diesem "Green Cabinet" gehören Staatssekretäre nahezu aller Fachministerien an. Hier sollen Ziele und Maßnahmen der Ministerien auf gegenseitige Beeinflussung hin betrachtet werden, um zu einer kohärenteren Politik zu kommen. Das Green Cabinet hat inzwischen drei Handlungsfelder - Verkehr, Energie und Landwirtschaft - benannt und für diese Projekte definiert, mit deren Hilfe der Weg zu einer nachhaltigen Entwicklung unter ökologischen $\mathrm{Ge}$ sichtspunkten geebnet werden soll (5). Allerdings fehlt den bisherigen Aktivitäten ein übergeordnetes Ziel und die entsprechende Gesamtstrategie für eine nachhaltige Entwicklung.

Auf Ebene der EU wurde bisher hingegen auf institutionelle Veränderungen verzichtet. Eine Notwendigkeit hierzu lässt sich möglicherweise nach Abschluss des Cardiff-Prozesses ableiten.

\section{Never Impuls durch Nachhaltig- keitsstrategien}

Auf europäischer wie deutscher Ebene wird an der Entwicklung einer Nachhaltigkeitsstrategie gearbeitet. Bis zur Konferenz "Rio+10" 2002 in Johannesburg müssen dringend die Hausaufgaben der Riokonferenz von 1992 erledigt werden, damit sowohl Deutschland als auch - oder vor allem - Europa mit gutem Beispiel für zögernde Industriestaaten, wie die USA und Japan, und Schwellen- wie Entwicklungsländer vorangehen können.

Im Juli 2001 stellte die EU-Kommission in Göteborg einen Vorschlag für eine europäische Nachhaltigkeitsstrategie vor (6). Die Handlungsfelder schließen sozial-ökonomische Bereiche ebenso ein wie die Nutzung der Natur als Ressource und Senke. Explizit genannt werden unter anderem 
die Bereiche Klimaschutz und Verkehr. Eingeschlossen sind zudem Vorschläge für institutionelle Veränderungen sowie die Überprüfung der gemeinsamen Politiken in den betroffenen Feldern. Der Europäische Rat hat den Vorschlag wenn auch in zu erwartender weicherer Formulierung - in vielen Bereichen angenommen und fordert die Mitgliedstaaten zur Entwicklung eigener Nachhaltigkeitsstrategien auf (7).

Auch in Deutschland werden erste Schritte auf dem Weg einer Nachhaltigkeitsstrategie gegangen. Gemeinsam mit dem Green Cabinet wurde die Einsetzung eines Rates für Nachbaltige Entwicklung beschlossen. Die Mitglieder wurden jedoch erst verspätet im Frühjahr 2001 benannt. Auch deren Auswahl ist nicht frei von Kritik. Sie spiegeln zwar durch VertreterInnen konstituierter Interessengemeinschaften unterschiedliche gesellschaftliche Gruppen wider, aber eben doch nicht alle (8). Eine der Aufgaben des Rates ist die Verbreitung und Rückkopplung der Nachhaltigkeitsstrategie und deren Handlungsfelder, Ziele und Maßnahmen in der Bevölkerung, doch steht ein Großteil der Bürger nicht in engem Kontakt mit den vertretenen Interessengruppen.

Die Vorlage eines Vorschlags zur Nachhaltigkeitsstrategie durch den Rat soll zum Jahresende erfolgen. Dieser Tage stellt er der Öffentlichkeit erste Arbeitsergebnisse vor, die er in den gesellschaftlichen Dialog einbringen will.

\section{- Die Rolle von Politikintegration}

Sowohl auf deutscher wie europäischer Ebene zeichnet sich die Verbindung von Nachhaltigkeitsstrategie als Programm und Politikintegration als Instrument ab. Während der Europäische Rat explizit die Fortführung des CardiffProzesses anmahnt und dessen Beitrag zur Umsetzung einer Nachhaltigkeitsstrategie benennt, wird die Verbindung in Deutschland eher unterschwellig über das Green Cabinet deutlich: Dieses hat nicht nur Arbeitsschwerpunkte und Projekte für eine nachhaltige Entwicklung definiert, sondern ist auch für die Abstimmung des vom Nachhaltigkeitsrat vorgelegten Strategieentwurfs zuständig.

Aus der bisherigen Erfahrung sollte deutlich geworden sein, dass eine nachhaltige Entwicklung ohne Politikintegration nicht erfolgreich eingeleitet werden kann. Auch die geläufige Definition der drei gleichberechtigten Säulen Ökologie, Ökonomie und Soziales spricht hierfür.
Dennoch orientieren sich Politikintegration und Nachhaltigkeitsstrategie in den aktuellen Stadien vor allem an ökologischen Belangen. Insbesondere im Handlungsfeld Energie sind in Deutschland von Rot-Grün viele Maßnahmen ergriffen worden, die diesen Ansatz rechtfertigen, da erste Erfolge im Sinne eines ökologischen Strukturwandels zu erkennen sind. Für andere Felder des Klimaschutzprogramms gilt dies hingegen nur begrenzt. Allein mit technischen Maßnahmen und ordnungsrechtlichen Vorgaben lässt sich kein ökologischer Strukturwandel erreichen. Hier kommt es zusätzlich auf umfassende Veränderungen von Institutionen, Entscheidungsprozessen und Verhalten im Rahmen eines umfassenden Konzeptes an. Dies gilt - neben dem Bereich Energie - verstärkt für das Handlungsfeld Verkehr, aber auch für den Bereich Landwirtschaft, bei dem der Gipfel öffentlicher Empörung und Sorge für erste Ansätze eines Strukturwandels genutzt wurden. Der Nachhaltigkeitsrat spielt bei der Festlegung der Handlungsfelder, Ziele und Maßnahmen für eine Nachhaltigkeitsstrategie eine wichtige Rolle. Ob es ihm gelingt, seine Ideen auch in die Gesellschaft rückzukoppeln, die Akzeptanz der entwikkelten Nachhaltigkeitsstrategie zu erreichen und so einen breit getragenen Strukturwandel voran-

VÖW-Schriftenreihe der AG Ökologischer Strukturwandel erschienen

Der jetzt vorliegende 40-seitige Band geht aus einer zwölfteiligen Artikelserie "Ökologische Modernisierung" in der Frankfurter Rundschau zwischen September und Dezember 2000 hervor. Die Einzelbeiträge wurden für die Schrifffassung zum Teil aktualisiert oder leicht verändert. Sie befassen sich zunächst mit Bedingungen, die für die erfolgreiche Umsetzung einer ökologischen Modernisierung zu schaffen sind, dem Instrument Ökologische Steuerreform und der Klimapolitik. Danach wird auf notwendige Änderungen beim Vorgehen des Akteurs Politik sowie die Rolle von Unternehmen, Konsumentlnnen und NGOs eingegangen. Abschließend wird das Zusammenspiel der Akteure in den Handlungsfeldern ökologisch-ethisches Investment, Verkehr sowie Hochschulen näher betrachtet. Der Band "Ökologische Modernisierung", ISBN 3-9807749-0-2, kann über die Geschäftsstelle der VÖW für 8 Mark (Mitglieder) bzw. 10 Mark (NichtMitglieder) bezogen werden: Vereinigung für ökologische Wirtschaftsforschung (VÖW) e.V., Potsdamer Str. 105, 10785 Berlin, Tel. 030/ 8851800, Fax 030/8825439, E-mail: info@voew.de zubringen, bleibt abzuwarten. Politisch festgelegte Ziele können nur erreicht werden, wenn sie auch von allen anderen Akteuren - etwa Industrie, Bürger, Interessenverbände, Wissenschaft und Forschung - nachvollzogen und unterstützt werden. Einen Strukturwandel ohne Veränderungen von Bewusstsein und Verhalten erreichen $\mathrm{zu}$ wollen, scheint jedenfalls nicht möglich.

Eine durchdachte und langfristig ausgerichtete Nachhaltigkeitsstrategie könnte den ökologischen Strukturwandel als Prozess und Bestandteil einer nachhaltigen Entwicklung befördern. Der Nachhaltigkeitsrat muss seinen Entwurf "nur noch" wegweisend in diese Richtung erstellen und das Green Cabinet überzeugen, diesen Weg zu gehen und nicht bei einer ökologischen Modernisierung als ersten Schritt stehen zu bleiben. So könnte durch Politikintegration ein Dominoeffekt angestoßen werden, der ökologischem Strukturwandel und Nachhaltigkeitsstrategie zum Durchbruch verhilft.

\section{Anmerkungen}

(1) Vgl. dazu Schepelmann, P.: Kollaps oder Rückkehr zur Politik? Auf dem Weg zu einer Nachhaltigkeitsstrategie der Europäischen Union. In: Ökologisches Wirtschaften, Nr. 2/2001, S. 5-6.

(2) Ecologic: Ergebnisse des "Cardiff-Prozesses" zur Integration der Erfordernisse des Umweltschutzes in andere Politiken - Bewertung des Zwischenstandes, Forschungsbericht (BMU/UBA) Nr. 29919 120; als Download unter http:// www.ecologic.de/deutsch/download/index.html.

(3) Vgl. hierzu ausführlich Volkens, A./ Büsgen, U.: Beendigung der isolierten Umweltpolitik durch Umweltintegration. In: VÖW (Hrsg.): Ökologische Modernisierung. Berlin 2001, S. 10-13.

(4) Das Programm im Internet unter www.bmu.de.

(5) Die Beschreibungen der Handlungsschwerpunkte und diesen zugehöriger Projekte finden sich unter http://www. bundesregierung.de/frameset/index.jsp oder unter www. nachhaltigkeitsrat.de/links/\#p2

(6) Mitteilung der Kommission KOM(2001)264 endgültig vom 15. Mai 2001.

(7) Schlussfolgerungen des Vorsitzes - Europäischer Rat, Göteborg, 15. und 16. Juni 2001.

(8) Siehe auch Nordbeck, R.: Ist langsamer wirklich nachhaltiger? In: Ökologisches Wirtschaften, Nr. 2/2001, S. 4.

\section{Die Autorin}

Annette Volkens ist Pressesprecherin der Vereinigung für ökologische Wirtschaftsforschung und eine Koordinatorin der AG Ökologischer Strukturwandel.

Kontakt: Annette Volkens, Tel. 030/ 3455449,

E-mail: annette.volkens@voew.de 
(c) 20I0 Authors; licensee IÖW and oekom verlag. This is an article distributed under the terms of the Creative Commons Attribution Non-Commercial No Derivates License (http://creativecommons.org/licenses/by-nc-nd/3.o/), which permits unrestricted use, distribution, and reproduction in any medium, provided the original work is properly cited. 